\title{
El ritual en los Textos de las Pirámides: sintaxis, texto y significado
}

\author{
Antonio J. MORALES \\ Freie Universität Berlin, Ägyptologisches Seminar \\ a.morales@fu-berlin.de
}

\section{RESUMEN}

Este trabajo pretende explorar la dimensión ritual en los Textos de las Pirámides, el corpus de literatura religiosa extensa más antiguo de la humanidad. La naturaleza variada de sus componentes textuales ha impedido que los egiptólogos comprendan en profundidad las complejidades de la colección y los contextos originales en los que estos textos (ritos) aparecieron. La aplicación de la teoría del ritual, principalmente la aproximación de la sintaxis ritual, ofrece a los investigadores un marco excelente de análisis e interpretación del corpus, su estructura y función. Sujeto a las reglas de la sintaxis ritual es posible exponer los múltiples niveles de significado en el corpus para la resurrección y salvación del difunto.

Palabras clave: Textos de las Pirámides, ritual, sintaxis, literatura funeraria, recitaciones, grupos, series, tradición, innovación, adaptación, Reino Antiguo.

\section{Ritual in the Pyramid Texts: syntax, text and meaning}

\begin{abstract}
This paper aims at the exploration of the ritual dimension in the Pyramid Texts, the earliest instance known to humankind of substantial religious literature. The variegated nature of the textual constituents in the corpus has prevented Egyptologists from understanding in depth the complexities of the collection and the original contexts in which these texts (rites) first emerged. The implementation of ritual theory to the corpus, particularly the syntax ritual approach, provides the scholars with an outstanding frame of analysis and interpretation of the corpus, its structure, and function. With the corpus anchored to the rules of ritual syntax, it is possible to expose its multiple layers of meaning for the deceased's resurrection and salvation.
\end{abstract}

Keywords: Pyramid Texts, ritual, syntax, mortuary literature, recitations, groups, series, tradition, innovation, adaptation, Old Kingdom.

SUMARIO: 0. Introducción. 1. Los Textos de las Pirámides: de lo general a lo particular. 2. Teoría del ritual y los Textos de las Pirámides. 3. Sintaxis y significado ritual. 4. Conclusión. 5. Referencias bibliográficas. 


\section{INTRODUCCIÓN ${ }^{1}$}

A finales de la Dinastía V -hacia el año 2345 a.C.- el rey Unas fue enterrado en su pirámide de Saqqara, la necrópolis en la que se habían enterrado los reyes de los últimos tres siglos. A diferencia de sus antecesores, Unas ordenó inscribir sobre los muros de su cámara funeraria una colección heterogénea de textos religiosos, conocidos hoy día como Textos de las Pirámides, cuyo propósito fundamental era perpetuar la celebración de los rituales funerarios y de ese modo asegurar la transformación del rey difunto en un espíritu todopoderoso, un akh (Allen 2005, 1-3; Hornung 1999, 1). El predominio de aspectos solares en el corpus sugiere que fueron sacerdotes menfitas y heliopolitanos quienes a mediados del Reino Antiguo recogieron, custodiaron y diseñaron la primera de estas colecciones para un rey, ${ }^{2}$ sin sospechar que estos materiales experimentarían una larga transmisión hasta los primeros siglos de nuestra era.

A pesar de haber identificado los diversos grupos y series que lo componen, el estudio del origen y naturaleza de este corpus ha generado interpretaciones de índole diversa. ${ }^{3}$ Inicialmente algunos investigadores creyeron que la selección de textos de cada pirámide reflejaba los ritos del funeral para su propietario (Altenmüller 1974; 1972; Spiegel 1971; 1956; 1953; Schott 1950, 153-154). Más tarde, otros especialistas entendieron que los textos de cada pirámide conferían un conocimiento mágico al rey para asegurar su travesía al Más Allá (Barta 1981, 66-69); e incluso algunos han interpretado los textos como literatura mitológica que servía al difunto como guía

${ }^{1}$ En el presente trabajo se han reducido lo más posible las abreviaturas comunes en la disciplina egiptológica: «TP» para las recitaciones individuales de los Textos de las Pirámides; «Pir.» para las líneas asignadas a cada recitación en Sethe 1908-1922; «TA» para el corpus de los Textos de los Ataúdes; y «ECT», acompañado un número ordinal, para los Textos de los Ataúdes en su edición en texto jeroglífico en Allen 2006a y De Buck 1936-1961. Los textos egipcios que han sido traducidos al castellano aparecen acompañados de su correspondiente transliteración para el especialista y muestran la fuente de origen, ya sea la pirámide de un rey del Reino Antiguo (i.e. Unas, Teti, Pepi I, Merenre, Pepi II) o una tumba, ataúd o pieza de ajuar funerario del Reino Medio. Para la nomenclatura de fuentes del Reino Medio (ejs. T9C, Sq18X), téngase en cuenta que la primera sigla indica origen ( $\mathrm{T}=$ Tebas; $\mathrm{Sq}=$ Saqqara), el número indica el orden de registro, y la última letra hace referencia a la localización actual ( $\mathrm{C}=$ Museo de Cairo; $\mathrm{X}=\mathrm{perdido}$ / destruido).

2 Para la formación de este corpus de textos «autorizados» bajo la influencia de la teología heliopolitana, véase Bickel 1994, 285-298.

${ }^{3}$ Los términos «grupo», «secuencia» y «serie» han sido utilizados con anterioridad en el estudio de esta composición, recientemente en Hays 2012, 79-81 (grupo) y 120-123 (secuencia). En general, una secuencia consiste en una conjunto de más de dos textos que aparece al menos en dos fuentes con los mismos textos constituyendo el conjunto y en el mismo orden (cf. Hays 2006, 6, 11-13, «recurring series»); y Altenmüller 1972, 40-51). La diferencia con una serie es que esta última presenta textos de este conjunto pero en distinto orden y con algunas modificaciones (incorporaciones de nuevos textos y ausencias de textos del conjunto original). Para el concepto de «serie», véase Hays 2012, 122, n. 491. El concepto de grupo fue discutido originalmente en Allen 1994, 38-39; y Altenmüller 1972, 46, aunque dichas descripciones han sido posteriormente criticadas por Hays 2012, 5, n. 32. Se entiende como grupo al conjunto de textos con afinidades temáticas y funcionales que, consecuentemente (pero no necesariamente), suelen aparecer en una misma serie o secuencia. 
ascensional (Hellum 2001, 34-42). En la actualidad se ha avanzado bastante en la comprensión de esta colección de textos, entendiendo que refleja rituales de diversa naturaleza que existían en el dominio de la oralidad mucho antes de que se transfieriesen al dominio escriturario en la pirámide de Unas.

Por ello, el principal factor que explica el funcionamiento de este corpus y su notoria transmisión es la heterogeneidad de sus constituyentes. Cada una de las selecciones atestiguadas de Textos de las Pirámides representa una sucesión de grupos de textos rituales con una secuenciación particular, adaptada a las prácticas y creencias de la tradición que se deseaba reflejar. Consecuentemente, en el proceso de transmisión del corpus los sacerdotes y escribas encargados de la composición de nuevos programas podían especular con los textos y enfatizar diversas doctrinas teológicas, creencias populares y, sobre todo, tradiciones rituales.

En este trabajo se pretende explicar los principales aspectos rituales en los Textos de las Pirámides a la luz de diversas teorías sobre el significado y la estructura del ritual. En particular se da importancia a la tendencia estructural que identifica el orden de los rituales con la sintaxis del lenguaje, una aproximación relevante si se tiene en cuenta que el corpus de los Textos de las Pirámides representa las voces y actos mismos de sacerdotes ejerciendo una serie de ritos que terminaron por monumentalizarse en forma de inscripciones apartadas del mundo, inicialmente en las paredes de las cámaras subterráneas de los reyes del Reino Antiguo, donde ningún individuo podría volverlas a leer nunca más.

\section{LOS TEXTOS DE LAS PIRÁMIDES: DE LO GENERALA LO PARTICULAR}

Los dos elementos fundamentales de la integridad narrativa de un corpus -antiguo o moderno- son su orden lógico y la relación interna de todas sus partes. En su conjunto, el corpus de los Textos de las Pirámides no responde a ninguno de los dos requisitos y, por lo tanto, carece de narrativa y de argumentos narrativos internos que encadenen sus distintos componentes. Sin embargo, su estructura alógica muestra un alto grado de intertextualidad, sobre todo por las continuas y numerosas repeticiones en el discurso. Son estas repeticiones las que definen el corpus como un conjunto sistemático de motivos rituales que debían perpetuarse en la pirámide con el único propósito de asegurar la resurrección del rey difunto y preservar la existencia de su espíritu en el Más Allá.

La cuestión principal es que el corpus no se originó como una obra para ser leída de principio a fin, sino que surgió por la combinación de ciertas prácticas cultuales y la voluntad de transmitir las voces y acciones de estas ceremonias a través de su monumentalización (Hays 2012; Billing 2011) . Por lo tanto, la aparición de la lite-

\footnotetext{
${ }^{4}$ En cuanto a este fenómeno, Hays 2012, 13, argumenta que «the rite did become an object of knowledge. This represented a crucial historical shift in Egyptian religious history. It was a question of the elevation of belief over practice (...) It is precisely in the context of a redirection of cultural interest away from the
} 
ratura funeraria egipcia en el tercer milenio a.C. se debe a un plan de adaptación de materiales orales a las eventualidades físicas y mediáticas de la epigrafía funeraria, una innovación aplicada por primera vez en las cámaras selladas del rey Unas. Como el contexto original del corpus no fue la pirámide sino un cúmulo de ceremonias de diversa índole, antigüedad y naturaleza, la obra resultante nunca adquirió una secuencialidad fija o una narrativa definida, pero transformó lo evanescente en permanente (Hays 2012, 114). Y lo que es más importante, la forma del corpus en cada pirámide del Reino Antiguo y en las versiones posteriores, en su complejo proceso de transmisión, vino a depender plenamente de la tradición funeraria y las prácticas rituales que reflejase; de ahí su flexibilidad, variabilidad y multiplicidad de formas (Morales 2013, 1-2).

Es este sentido, el argumento de que los rituales, como producciones iterativas, tienen la misma estructura formal o sintaxis que los idiomas naturales es extremadamente significante (Seaquist 2004, iv; Staal 1995, 1-6). En el Egipto antiguo -como en otras civilizaciones- la estructura de los rituales siguió un orden similar al del lenguaje en el que todas las partes presentaban conexiones semánticas. Esto significa que los rituales egipcios y su expresión a través de los textos eran tan vulnerables a alteraciones como el lenguaje ritual que expresaba las propias ceremonias. En base a esta relación, cualquier transformación de una ceremonia implicaba la redefinición del lenguaje a través del cual el discurso ritual se expresaba y, consecuentemente, de la articulación textual de dicho rito. La mecánica de este principio también podía funcionar a la inversa: la forma ritual era susceptible al cambio si se producía la transformación del lenguaje y los textos que la expresaban. Sin importar el origen o dirección del cambio en cuestión el efecto era el mismo (ritual $\leftrightarrow$ lenguaje $\leftrightarrow$ texto): la modificación de una de las dimensiones del ritual afectaba a los otros dos elementos del fenómeno (Gruenwald 2003, vii; Greimas $1983,21-22)^{5}$.

La configuración y ductilidad de los Textos de las Pirámides no sólo justifican la transmisión constante de sus materiales desde el Reino Antiguo hasta la época Grecorromana, sino que además explican los principios esenciales del propio corpus y del marco de la literatura funeraria al que pertenece:

1) El corpus está constituido por textos de carácter ritual que se dividen en dos categorías fundamentales (Hays 2012, 17-45; Roeder 2004, 20-36) ${ }^{6}$ : textos sacerdotales (i.e. recitaciones en las que un oficiante ejecuta los rituales para

\footnotetext{
operative deed over to the idea that the mortuary literature tradition was born».

5 Sobre la sintaxis de un ritual, en términos generales, Gruenwald 2003, vii, afirma que «an important principle of rituals is that the components of the ritual act require an orderly performance. This order establishes the inner logic of the ritual acts».

6 Para la distinción de los dos tipos de categorías basándose en su deixis, véanse Hays 2012, 31 (n. 2); 2009, 49 (n. 15); Allen 1994, 16-18; Assmann 1990, 14; Barta 1981, 65-67. Para la importancia de las «expresiones indexicales» en la regulación del discurso ritual, véase Hanks 1992, 43-76.
} 
el difunto) ${ }^{7}$ y textos personales (i.e. recitaciones en las que el difunto es su propio oficiante) $)^{8}$.

2) El corpus no incluye episodios narrativos extensos de carácter mitológico sino referencias o nociones míticas oblicuas, «mitemas» ${ }^{9}$, que reflejan la existencia de construcciones cosmogónicas (Molinero 1999, 17).

3) De las dos categorías de textos existentes en el corpus (sacerdotal y personal), el grupo de textos sacerdotales experimenta una transmisión más continuada (Hays 2012, 14; 2009, 49; 2006, 31-32). Este factor evidencia el carácter ceremonial del conjunto y la discontinuidad del grupo de textos personales, sustituidos por otras composiciones similares (i.a. Textos de los Ataúdes y Libro de la Salida al Día).

4) Es concebible -y verificable- que la multiplicidad de compilaciones atestiguadas para los Textos de las Pirámides en las diversas necrópolis del país desde el Primer Periodo Intermedio (Dinastías IX-X) hasta la Época Baja (Dinastías XXV y XXVI)- responde a la adaptación del corpus a las tradiciones cultuales funerarias (i.e. ritual) y, en menor medida, al pensamiento teológico de cada localidad (Morales 2016a). La simple identificación de varias tradiciones textuales en un mismo centro es suficiente para invalidar la llamada «teoría de los regionalismos», una propuesta que ha explicado las diversas formas del corpus como el resultado de la especulación teológica y mitológica de los sacerdotes y escribas de cada región (Hoffmeier 1996; Silverman 1989)

\footnotetext{
${ }^{7}$ Ejemplo de texto de la categoría sacerdotal: TP 197 Pir. 113a-b (Pepi II)

$\underline{d} d-m d w$

Wsjr Ppj

$j r(. t) \operatorname{Hr}$ tn $r w \underline{d} . t d(=j) n=k s(j)$

jmjm $=k$

$n r n=k$ hftj $=k$

$t d w 3 m-\underline{d} r=f$

${ }^{8}$ Ejemplo de texto de la categoría personal: TP 227 Pir. 227a-c (Unas) $\underline{d} d-m d w$

hsq(:j) $m$ tp $\mathrm{k} 3 \mathrm{~km} w \mathrm{r}$

hpnw $\underline{d} d(=j)$ nn $r=k$

hsr-ntr $\operatorname{srq}(. t) \underline{d} d\left({ }^{\prime} j\right)$ nn $r=k$

$p n ' \underline{t w} h b h(n)\left[t_{3}\right]$

dd.n(:j) nn $r=k$

$d d . n(=j) n n r=k \quad$ porque yo he dicho esto contra ti.
${ }^{9}$ Véanse las referencias oblicuas en, $\mathrm{p}$. ej., TP 468 Pir. 895a-d (Pepi I)

h3 Ppj pn $\underline{t} z \underline{t} w$ ' $h$ '

jp.n tw ps d.t' $3 . t$ jm.t Iwnw

jr s.t $k$ wr.t

hms $=k$ Ppj pw hnt ps $\underline{\text { d.t }}$

Gb js jrj-p'(.t) ntr.w

Wsjr js hnt shm.w

Hr js $n b$ p'.wt ntr.w

Recitación:

¡Osiris Pepi (Neferkare)!

En cuanto a este Ojo de Horus firme, yo te lo entrego para que seas fuerte

y para que tu enemigo se asuste de ti.

Pan de la mañana frente a él.

Recitación:

¡Yo he cortado la cabeza del gran toro negro! ¡Oh serpiente Hepenu, yo digo esto contra ti!

¡Oh repulsivo escorpión, yo digo esto contra ti!

Vuélvete, introdúcete en la tierra,

¡Oh Pepi, álzate, ponte en pie!

La gran enéada que está en Heliópolis te ha asignado

a tu gran trono

para que tú, Pepi, puedas sentarte al frente de la enéada,

como Gueb, un líder entre los dioses,

como Osiris a la cabeza de los poderes,

y como Horus, señor de los hombres y los dioses.
} 
y no como un proceso adaptativo del corpus de textos a las diversas tradiciones rituales del país (Morales 2016b; 2013).

5) En general, la mayoría de las recitaciones que conforman el corpus de los Textos de las Pirámides manifiesta cierta relación con las prácticas y creencias funerarias del contexto privado ${ }^{10}$, tanto en los orígenes del conjunto (Morales 2016b; 2015a) como en su desarrollo durante el Reino Antiguo (Hays 2012) o en el ámbito coetáneo de las inscripciones funerarias privadas, como en los casos de Khesu de Kom el-Hisn, Pepy-Ima de Mendes, Anu de Saqqara y Medunefer de Balat (Silverman 1988; 1996; Russo 2012; Valloggia 1986). A pesar de que sus textos han sido etiquetados como Textos de los Ataúdes, en la mayoría de los casos estos materiales proceden de los mismos dominios cultuales de los que sacerdotes y escribas menfitas seleccionaron el compendio de recitaciones para los reyes de la V y VI Dinastías. Por lo tanto, estos textos obedecen a los mismos propósitos del corpus para el monarca pero pretenden la resurrección y salvación de cualquier difunto (Morales 2013; Hays 2012, 2; Mathieu 2004).

Llama la atención que de las propiedades expuestas para el corpus de Textos de las Pirámides se haya minimizado su aspecto mitológico. Un análisis exhaustivo de los materiales que lo componen justifica la vertiente ritual, performativa y perlocutiva $^{11}$ que se desvincula de la existencia de relatos narrativos extensos en el Reino Antiguo (Assmann 1977, 7-43; contra: Altenmüller 1999; Kahl 1999; Zeidler 1993). Una de las razones para enaltecer la función ritual sobre la mítica en este corpus es evidente: además de carecer de consistencia narrativa, el corpus refleja una doctrina de la resurrección aplicada a un individuo genérico, i.e. cualquier rey para quien se personaliza el conjunto de recitaciones con su nombre, y no un monarca ficticio o pseudo-histórico (Hays 2012, 8). En cuanto a las referencias míticas oblicuas del texto, Hartwig Altenmüller explica su funcionamiento sólo como complemento del acto ritual $^{12}$, para la adecuada transposición del rito al mundo de los dioses $(1972,74)$.

\footnotetext{
${ }^{10} \mathrm{Cf}$. el listado sucinto de ritos de ofrenda (i.e. mención exclusiva del objeto de ofrenda) en la estela privada de la III Dinastía de Khabausokar (TP 113, TP 32, TP 25, TP 77, TP 74, TP 154, TP 177, TP 89, TP 142, TP 154, TP 160) y la lista de ritos de ofrenda de los Textos de las Pirámides de Unas (TP 23, TP 25, TP 32, TP 34-42, TP 32, TP 43-57, TP 72-79, TP 81, TP 25, TP 32, TP 82-96, TP 108-171). Por exponer uno de estos ritos y ejemplificar cómo se expresaban, véase cómo se estipula el rito TP 154 para Khabausokar (jrp ‘b̌ 2 «dos jarras de vino») y para el rey Unas (Wsjr Wnjs $m-n=k$ jr.t Hrw bšt.n=sn hw ' $m=f$ s jrp 'bš 2 « Oh Osiris Unas! Toma para ti el Ojo de Horus que ellos escupieron y evita que él se lo trague. Dos jarras de vino»).

11 Véanse exhortaciones, imperativos, mandatos, p. ej., en TP 419 Pir. 747a-748b (Teti)

wn '3.w hrr..jw) št3.w s.wt

'h'j.dr t3=k why hm.w=k $\underline{t} z \underline{t} w$

hnzz=k $m$ 'b 3 hj.w

$\underline{d} n h w j=k m$ bjk $z j \check{j} \tilde{j}=k m$ s $b_{3}$

12 Por ejemplo, TP 34 Pir. 26a-f (Unas)

zmjn zmjn wpp $r=k$

h3 Wnjs j.dp=k dp.t=f hnt zh-ntr

Que las puertas de los que controlan los sitios inaccesibles se abran. ¡Levántate! ¡Quítate tu tierra! ¡Sacúdete tu polvo! ¡Ponte en pie! Que puedas viajar en compañía de los espíritus akh, tus alas como un halcón (y) tu resplandor como una estrella.

¡Crema zemin, crema zemin que abre tu boca!

¡Oh Unas, probarás su sabor frente a la tienda del dios!
} 
De este modo, la aproximación presente desiste de seguir la tendencia de la Escuela de Cambridge que entiende el ritual como complemento del mito (Segal 2006; 1980; Strenski 1996; Ackermann 1991; Doty 1986, 73-78; Kirk 1973, 61-69; 1970, 16-31) y no lo concibe en sí como una estructura independiente con sus propias reglas y principios. A pesar de la relación entre el ritual y el mito en los Textos de las Pirámides, la función principal de las recitaciones inscritas en las cámaras funerarias ocultas era la de perpetuar los actos ceremoniales para la resurrección del monarca y no la de reflejar cierta correspondencia entre la realidad y la esfera mítica de lo divino (pace Hellum 2014; 2001; Mercer 1956). Eso significa, por ejemplo, que no se puede adoptar el enfoque clásico de Mircea Eliade y su escuela, que defienden la función del ritual sólo como una recreación del mito (Allen 1978, 105-200; Bleeker 1972; Eliade 1957, 9), reduciendo el evento performativo y perlocutivo a una simple articulación de tintes cosmogónicos y dramáticos (cf. Gillam 2005; Ritner 1997, 35-53).

El sistema de prácticas funerarias en el Egipto antiguo no acoge bien esta interpretación ya que ciertas ceremonias no persiguen relacionar el acto mismo con una construcción mítica concreta o recrear un mito, sino que buscan formalizar el papel del oficiante y progresar de lo terrenal a lo divino para ofrecer potencia mágica al difunto. Así, por ejemplo, en el ritual de la excavación de canales representado en la paleta y la maza ceremonial del rey Escorpión (II) de época predinástica se observa que el evento enfatiza el rol creador del monarca, implica el acto fundacional primordial y asocia la acción del rey con el mundo de los dioses (Baines 1995, 9; Goldwasser 1992,71-72). De hecho, aunque esté presente, la referencia mítica que podría sustraerse de este evento - la identificación del rey con un dios demiurgo que crea y ordena el mundo- no constituye la intención primera de su representación (Morales 2016c; Tobin 1992).

La cuestión de la existencia del mito en el Reino Antiguo, estudiada a través del corpus de los Textos de las Pirámides, ha dado lugar a un fuerte debate. Recientemente, Jennifer Hellum ha enfatizado que se ha pasado de discutir si existieron los mitos en el Reino Antiguo a cómo existieron los mismos, haciendo hincapié en la existencia de un metamito reflejado a través de la combinación de la arquitectura piramidal, los textos rituales y las referencias míticas (Hellum 2014, 124) ${ }^{13}$. En 1945 Siegfried Schott ya señaló una clara predominancia de los textos rituales sobre las nociones míticas del corpus (Schott 1945), pero ha sido Jan Assmann quien ha propuesto que la

\footnotetext{
jššw hr zmjn ǰ̌šw Stš zmjn

tt jb nb.wy zmjn

$\underline{d} d-m d w z p 4$

$h z m n=k m$ 'b šmsw-Hr

bd Nhb Šs 'w $\underline{t}^{3} 5$

Horus escupe la crema zemin (y) Seth escupe la crema zemin.

Lo que reconcilia a los dos dioses es la crema zemin.

Recitación cuatro veces:

«Tú has sido purificado con los seguidores de Horus».

13 Véase, por ejemplo, las referencias espaciales en TP 364 Pir. 616d-f (Merenre)

rdj.t $n$ mw.t $k$ N Nwt $m$ rn=s $n \underline{d} r . w t$

jnq. $n=s \underline{t} w$ m $r n=s \quad n$ qrsw

$j . s j ' t j$ ins $m$ rns $n j$

[...] siendo entregado a tu madre Nut en su nombre de 'sarcófago', ella te ha abrazado en su nombre de 'ataúd' porque tú has sido elevado hacia ella en su nombre de 'tumba'.
}

'Ilu. Revista de Ciencias de las Religiones

2015, 20, 137-164 
existencia de mitos se debe relacionar con la presencia de relatos narrativos amplios, los cuales no se atestiguan en Egipto hasta el Reino Nuevo (ca. 1550-1069 a.C.). En su opinión, nociones como la gestación del rey por los dioses Gueb y Nut (resp. tierra y cielo) $)^{14}$, la progresión del dios Re por el firmamento ${ }^{15}$ o la ascensión del rey difunto en forma de estrella imperecedera (ikhem-sek) ${ }^{16}$ no podían considerarse construcciones míticas complejas y, por lo tanto, quedaban reducidas al nivel de sencillas explicaciones cosmogónicas (Assmann 2004, 31-57). Sin embargo, los defensores de la existencia de construcciones míticas sustanciales en el Reino Antiguo han argumentado que el componente mítico estaría profundamente asociado a la oralidad y las creencias populares, con alusiones míticas sucintas en los Textos de las Pirámides (Hellum 2012, 41-46; Stewart 2012, 197; Goebs 2003, 27-59; Eyre 2002, 137-152; Baines 1991, 81-105).

\section{TEORÍA DEL RITUAL Y LOS TEXTOS DE LAS PIRÁMIDES}

Dado que uno de los objetivos de este trabajo es enfatizar que los estudios de religión egipcia deben usar teoría de manera más prominente ${ }^{17}$, se incluye una discusión de las implicaciones teóricas del análisis de los Textos de las Pirámides y su estructura no-narrativa. Obviamente, como egiptólogo el objetivo principal que pretendo no es criticar las teorías antropológicas y religiosas del ritual, ni siquiera proponer alternativas conceptuales, aunque sí pueda afiliarme con alguna en particular. El propósito principal de esta sección es establecer una relación lógica entre este corpus de textos rituales y el marco teórico que se ha venido desarrollando en el estudio de las religiones antiguas (Kreinath, Snoek y Stausberg 2006; Bremmer 1998; Bell 1997), de modo que se presenten al lector los principios y convenciones que definen el ritual

14 P. ej. TP 366 Pir. 626a-627a (Teti)

$h_{3} W$ sjr Ttj 'h't $\underline{t} \underline{t} w$

$m s . n \underline{t w} m w . t=k$ Nwt sk.n $n=k$ Gb $r=k$

j.n $\underline{d} \underline{t} w$ ps $\underline{d} . t$ ' $3 . t$

dj. $n=s n n=k$ hft. $j=k \underline{h} r=k$

f3 $n=k$ wr jr=k j. n=sn jr=f

${ }^{15}$ P. ej. TP 606 Pir. 1687a-1687c (Merenre)

$h 3 w=k r=k m$ wj $p w n R$

mrrw ntr.w $j$ ' $n=f$

mrrw ntr.w h3.t jm=f

hnnw $R^{\prime} j m=f j r$ jh.t

h3w $M r-n-R^{\prime} j m=f R^{\prime} j s$

16 P. ej. TP 432 Pir. 782c-e (Pepi I)

t3 $\underline{h} r=\underline{t} r \underline{d} r=f j \underline{j} . n=\underline{t} s w$

$\check{s} n . n=\underline{t} n=\underline{t}$ t3 h.t $n b m \underline{h n w}$ '.wy=t

d. $n=\underline{t} n=\underline{t}$ Ppj pn $m$ j.hmm-sk jmj $\underline{\text { t }}$

d. $n=\underline{t} n=\underline{t}$ Ppj pn $m$ j.hnm-sk jmj=t $\quad$ y has emplazado a este Pepi como una estrella imperecedera en ti. aplicación de teoría a los datos empíricos de sociedades antiguas es un «scholarly desideratum».

¡Oh Osiris Teti! ¡Levántate, ponte en pie!

Tu madre Nut te ha alumbrado, Gueb te ha adecentado tu boca.

La gran enéada te cuidará,

habiendo puesto (ya) a tu enemigo a tus pies.

«Lleva contigo a uno que es más grande que tú», le dicen.

Tú subirás a bordo de esta barca de Re,

a la que los dioses desean acercarse,

a bordo de la cual los dioses esperan subir,

en la que Re es embarcado hacia el horizonte,

y en la que tú, (Nemtiemzaf) Merenre, te subirás como Re.

La tierra entera está bajo tu control pues tú la has conquistado.

Tú has abarcado la tierra y todas las posesiones con tus brazos, 
funerario egipcio a través de este corpus. Desafortunadamente, pocos egiptólogos han trabajado en este sentido (Roeder 2011; Te Velde 2003) ${ }^{18}$.

Los Textos de las Pirámides no solo son el corpus de literatura funeraria más antiguo de la humanidad sino también un medio para adentrarse en las tradiciones sociales y religiosas de una cultura milenaria y un conjunto empírico al que aplicar la teoría del ritual (Rappaport 1999, 32-50) ${ }^{19}$. En el Egipto del Reino Antiguo la práctica del ritual estaba firmemente definida por la estructura social y las restricciones que las normas y el decoro religioso imponían (Assmann 2000, 20-28; Baines 1990, 17-21). Sin embargo, la distinción moderna de las prácticas rituales para la realeza y la comunidad no refleja la verdadera relación de los dos ámbitos y las influencias comunes o permeabilidad observadas en sus textos y tradiciones. Como resultado, en la literatura egiptológica tradicional se han separado ambos contextos y desligado las composiciones para los reyes (Textos de las Pirámides) de aquellas que surgieron un poco más tarde para la elite (Textos de los Ataúdes), ignorando que en realidad ambas producciones eran el resultado de una misma tradición textual funeraria (Morales 2013; Mathieu 2004).

Por un lado, inicialmente, el acceso de la elite burocrática y religiosa a los Textos de las Pirámides desde la VI Dinastía se interpretó como una asimilación del privilegio exclusivo de la realeza a una existencia post mórtem (Kees 1952; Breasted 1933; 1912, 272; Sethe 1931; Moret 1922), una deducción incorrecta que ha provocado que este equívoco se mantenga hasta nuestros días (e.g. Picardo 2007, 226; David $2002,154)$. En realidad, desde las primeras dinastías el sistema egipcio de creencias y prácticas rituales garantizaba a todo individuo una experiencia de ultratumba. Ciertas transformaciones sociales, culturales y políticas del Primer Período Intermedio (Willems 2014) son las que permitieron un cambio en los medios de expresión para estas nociones escatológicas, adaptando el corpus de Textos de las Pirámides a las creencias ya existentes entre los miembros de la elite (Hays 2011, 115-130; Smith 2009, 1-16; Mathieu 2004, 256-258; Nordh 1996, 168-172; Silverman 1995, 80-81). A pesar de las limitaciones impuestas por las normas sociales y el decoro religioso, desde mediados del Reino Antiguo oralidad y escritura - esta última más restrictivamente (Baines 2004; 1999a; 1999b)- coexistían en el ámbito privado y eran el vehículo de expresión de creencias y tradiciones. Además, la iconografía jugaba un papel fundamental en la declaración de las ideas de la elite desde muy pronto (Silverman 2011;

\footnotetext{
18 Véase la crítica en Platvoet 1983, 177: «In a survey of the study of Egyptian religion in the Netherlands, D.J. Hoens recently quoted with apparent approval a statement by C.J. Bleeker in which it was argued that most Egyptologists had failed to give a satisfactory description of ancient Egyptian religion by their neglect of its rites (Hoens 1982: 19). Bleeker reproached his fellow-Egyptologists for not studying it from the point of view of the ancient Egyptians living in an agrarian society (i.e. from the emic or participant's point of view), but from that of modern Europeans who live in an industrialized society».

${ }^{19}$ Como indica Catherine Bell $(1997,235)$, «rituals do not build community by simply expressing sentiments of collective harmony; they do it by channeling conflict, focusing grievances, socializing participants into more embracing codes of symbolic behavior, negotiating power relations, and (...) forging images by which the participants can think of themselves as an embracing community».
} 
Baines 2010; 1994; 1989). Son evidentes las expresiones de rituales en las fuentes predinásticas y dinásticas tempranas, en las que los registros de escritura e iconografía dejaban entrever prácticas vinculadas a la expresión del poder real (Anderson 2011; Campagno 2003; Menu 2001) así como ritos de enterramiento y las primeras momificaciones (Tamorri 2012; Köhler 2003).

Por otro lado, la multiplicación de las tradiciones rituales tras el Reino Antiguo reguló la transmisión de grupos particulares de Textos de las Pirámides (sobre todo sacerdotales), que ahora se configuraban según las tendencias cultuales de cada cementerio y templo local. En consecuencia, en este trabajo se entienden las prácticas y textos rituales del período en el marco de una definición operacional (Platvoet 1995, 25) que postula la relación directa de las composiciones con los ritos celebrados. Esta definición justifica que la adopción y adaptación de la tradición textual se basase en la especulación religiosa a nivel editorial y ritual.

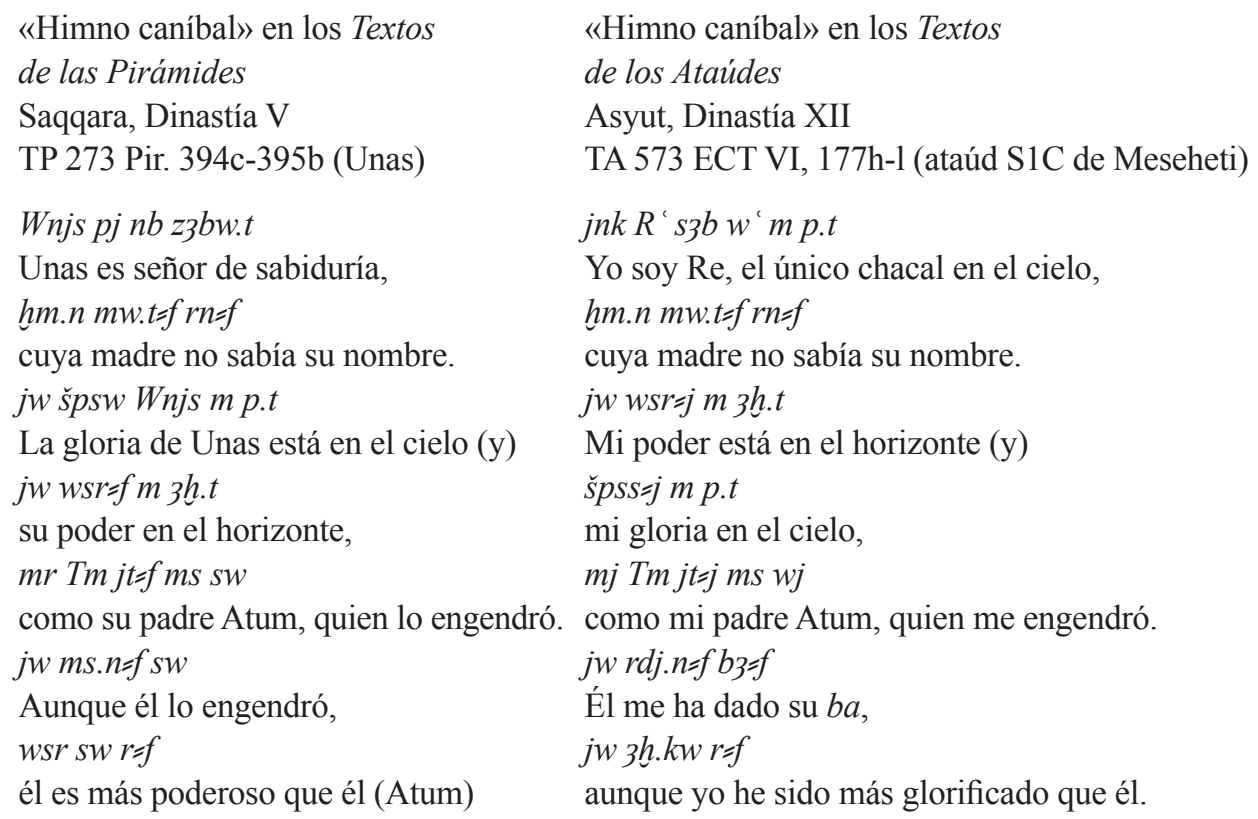

Según las palabras de Carl Andrew Seaquist, para determinar la clase de ritual conviene identificar la categoría del rito que se observa y el tipo de recitación que lo expresa. Como apunta Seaquist, «two components are, therefore, necessary to such a definition: first, the larger category (behavior) to which all rituals belong, and second, a character possessed by all rituals but by no other elements of the larger category» (Seaquist 2004, 40-43). En nuestro caso, los Textos de las Pirámides constituyen un collage ritual con dos categorías bien diferenciadas y varios grupos o series de diversa índole en cada una de las dos. Las dos categorías mencionadas anteriormente son la sacerdotal y la personal (Hays 2012, 10-13; 2009, 49). Mientras, la identificación de grupos con características comunes es más compleja debido a su elevado número: 
textos de ofrendas ${ }^{20}$, textos de resurrección ${ }^{21}$, textos de protección ${ }^{22}$, textos de transformación ${ }^{23}$, lamentos, apotropaia, etc. (Hays 2012, 79-111).

Sin embargo, en el estudio de la transmisión de los Textos de las Pirámides se observa que la distinción inicial de dos categorías se vio alterada por tres factores: 1) la transmisión más eficaz de textos sacerdotales; 2) la discontinuidad de muchos de los textos personales; y, 3) dejando una marca más profunda, la aparición de textos de nueva composición (Textos de los Ataúdes) y la integración de los Textos de las Pirámides en este nuevo repertorio textual. Estas modificaciones, sumadas a la emergencia de numerosas tradiciones regionales, gestaron la heterogeneidad característica de la literatura funeraria del Reino Medio.

El antropólogo Stanley Tambiah hizo uso de una aproximación similar (Tambiah 1979). En su estudio del ritual, Tambiah apuntó que el análisis de los ritos debía caracterizarse por su «openness to and search for patterns and rules of combination, sequencing, recursiveness, and redundancy that make up the totality, and that cannot be discovered by a too formulaic addition» (Tambiah 1979, 1). En este sentido, la noción del ritual y de los textos como una secuenciación redundante -no-narrativaes un concepto clave para la comprensión de la mecánica de trasmisión de los Textos de las Pirámides. Precisamente la combinación de numerosas series o secuencias de naturaleza diversa permitía al corpus su maleabilidad, adaptación y ajuste a las

\footnotetext{
${ }^{20}$ Ejemplo de texto de ofrenda: TP 77 Pir. 52a-c, 53b (Unas)

mrḥ.t mrh.t thij $w n=\underline{t}$

jm.t ḩ3.t Hrw thj wn=t

wn=t $m$ h3.t Hrw

dd( $=j) \underline{t} m$ m ḩ3.t Wnjs pn

snd $m=\underline{t} n=f \underline{h} r=\underline{t} s 3 \underline{h}=\underline{t} s w \underline{h} r=\underline{t}$

h.jt.t '̌s

${ }^{21}$ Ejemplo de texto de resurrección: TP 367 Pir. 622a-623a (Teti)

$\underline{t z} \underline{t} w$ Ttj $p w$ wn tw's phtj

hms $=k$ hnt $n \underline{t}$ r. $w$ jr=k $n w$

jr.n Wsjr m ḥw.t 'h'jm.t Iwnw

šzp $n=k s \grave{h}=k$

$n j \underline{d} r$ rd $=k m$ p.t $n j$ hssf=k $m$ t3

$n \underline{t} w t$ js 3 hj $m$ ms Nwt snqw Nb.t-hw.t porque eres un espíritu akh a quien Nut engendró y Neftis amamantó

${ }^{22}$ Ejemplo de texto de protección: TP 588 Pir. 1607a-1608a (Merenre)

Wsjr Mr-n-R 'pšš.n s mw.t=k Nwt hrr=k ¡Oh Osiris Merenre! Tu madre, Nut, se ha extendido sobre ti $m$ rn=s $n$ Stp.t

$r \underline{d j} . n=s$ wn=k $m$ ntr $n$ hft.j.j=k

$m r n=k n n \underline{t r}$

šnm.n=s kw m 'h.t $n b \underline{d} w . t$

$m$ rn=s $n$ hnmt-wr.t

${ }^{23}$ Ejemplo de texto de transformación: TP 309 Pir. 490a-491b (Unas)

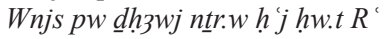

ms.n nh.t ntr.w

jm.t h3.t wj3 $R$

hms Wnjs $m$ b3h $=f$

j.wn Wnjs hn.w=fj.s $\underline{d}$ Wnjs wd. $w=f$

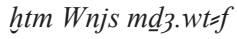

¡Ungüento, ungüento! ¿Dónde deberías estar?

Tú que estás en la frente de Horus, ¿dónde deberías estar?

Tú has estado sobre la frente de Horus,

yo te colocaré sobre la frente de este Unas.

Llevándote, tú deberías calmarlo y transformarlo en un espíritu $a k h$.

¡Levántate, Teti! ¡Apresúrate, tú grande de fuerza!

Tú te sentarás a la cabeza de los dioses y harás aquello que Osiris ha hecho en el recinto del mástil que está en Heliópolis. Ahora que tu dignidad te ha sido concedida,

en su nombre de 'Shetpet'.

Ella ha hecho que seas un dios para tu enemigo

en tu nombre de 'dios'.

Ella te ha protegido de todo acto malvado en su nombre de la 'gran protectora'.

Unas es el secretario de los dioses a cargo del gabinete de Re, nacido de aquella a quien los dioses imploran,

que está en la proa de la barca de Re.

Unas se sentará delante de él.

Unas abrirá sus cofres, romperá sus sellos,

y sellará sus documentos.
} 
formas locales de tradición funeraria. Por lo tanto, para la comprensión de la mutabilidad del corpus, del ritual, su sintaxis y divisiones se debían relacionar acción, recitación y texto. Esta relación entre rito y texto se hace palpable en el enfoque de Frits Staal sobre la sintaxis ritual. En cierta medida, éste es similar a la preocupación «estructural» percibida en los primeros trabajos de Noam Chomsky -principalmente en Syntactic Structures (1957)- donde los cambios gramaticales son el concepto clave para comprender las transformaciones de los diferentes miembros de una estructura (lingüística).

Sin embargo, más allá de la sintaxis o estructura que se pueda reconocer, uno de los problemas principales en el estudio de los rituales es intentar definir el propio acto ritual. Muchos especialistas han advertido que el número de definiciones que han aparecido hasta la fecha es interminable y que cada investigador cree poseer la mejor definición para este término (Humphrey and Laidlaw 1994). El ritualista Jan Snoek sugiere que todas las definiciones de ritual propuestas aspiran a precisar la noción por medio de formas lógicas clásicas (Snoek 2006, 3). Del mismo modo, Allan Grapard argumenta la aplicación de nociones occidentales al estudio de los rituales, por ejemplo de las sociedades y culturas asiáticas, las cuales difieren de las europeas y generan sus propios conceptos (Grappard 1991, 211-212). En relación con sistemas más alejados culturalmente, como la literatura funeraria egipcia, el corpus de textos del Reino Antiguo y Medio, incluyendo no sólo los Textos de las Pirámides y Textos de los Ataúdes sino también las inscripciones en tumbas y otros géneros relacionados, reflejan la historia de la ideología cambiante del Más Allá (Willems en prensa; Allen 2006b; Kákosy 1962; Anthes 1954, 25-27), del desarrollo de la tradición funeraria (Alexanian 2006, 8; Roth 1993, 48-55), del pensamiento escatológico y de las prácticas rituales cuya representación y significados se mantienen en la conciencia colectiva (Bakker 1993). Obviamente, tales significados no encuentran una correspondencia clara en nuestra sociedad y nuestra manera de concebir el mundo, por lo que debemos ser incluso más prudentes en nuestra interpretación y comprensión de la naturaleza del ritual en sociedades como la faraónica del tercer milenio a.C.

Los acercamientos generales a la definición del ritual divergen desde descripciones imprecisas (criticado por Goody 2010), meras ceremonias (Schmitz 1981, 163) y actos regulados por la comunidad (Van Gennep 1960; contra: Hays 2013) hasta incluso ritos no religiosos ${ }^{24} \mathrm{y}$ acciones carentes de todo significado (Staal 1989; 1981; $1980 ; 1979)^{25}$. En palabras de Richard Schechner, «ritual has been so variously

\footnotetext{
${ }^{24}$ Un ejemplo de rituales no religiosos puede encontrarse en en listado de ceremonias del Partido Nazi para conmemorar la sociedad alemana: véase Weitzel 1942, 8-9: »the celebrations of life-cycle (...) Birth Festival, Marriage Festival, and Festival for the Dead (...) must be conducted in good form and solely in accordance with the world-view of National Socialism, which is called to regulate the entire life of German people».

${ }^{25}$ En cierto modo una aproximación relacionada puede verse en Klingbeil 2007, 55, quien comenta que «[t]he actions that constitute a ritual do not have inherent meaning; rather, the meanings are determined by the cultural and religious context in which the ritual is enacted». Véase también Penner 2002, 153-155.
} 
defined as concept, praxis, process, ideology, yearning, religious experience, and function that it means very little for the simple reason that it can mean too much» (Schechner 1985, 192) ${ }^{26}$.

En algunos casos, como en los estudios de Jack Goody, se sugiere que los rituales poseen un significado particular. Esta consideración, aunque elemental, contradice otras posiciones como la de Frits Staal y su acentuación del acto sin significado. Goody explora esta premisa con la evaluación del impacto que tendría sobre una comunidad la ausencia de significado en un ritual de enterramiento, preguntándose cómo sería un funeral en el que no se hiciera referencia a la salvación o la vida tras la muerte (Goody 2010, 224). Recelando de la famosa tesis de Staal sobre el sin sentido del acto («meaninglessness»), la cuestión nos lleva a considerar que rito y ritual son conceptos cargados de semántica religiosa, social y cultural, sin mencionar las composiciones textuales que los representan y transmiten. En cierta medida, uno debe considerar que Staal comprendía la intentio ritus como subordinada al principal propósito del ritual, que era existir en sí mismo y ser ejecutado, y que el significado unido al ritual era solamente una interpretación post facto (Strenski 1991, 207-208; Staal 1989, 110).

El ritual de los Textos de las Pirámides y su estructura no pueden caracterizarse como «meaningless» por el simple hecho de que el corpus adquiere formas concretas en relación con cada una de las tendencias teológicas y ceremoniales del Reino Antiguo (Hays 2012), las tradiciones locales del Reino Medio (Morales 2015b; 2013), sus usos particulares en el Reino Nuevo (Hays and Schenk 2007, 97-115) o su adaptación a nuevas formas cultuales en Época Baja (Morales 2016a; Molinero et alii 2012). Por lo tanto, para que estas definiciones sean eficaces, en el estudio de los Textos de las Pirámides se debe promover el trabajo con las formas particulares de los grupos, series y textos, así como la estructura o sintaxis que representan en cada repertorio (i.e. en cada pirámide, tumba, ataúd, sarcófago, etc.) ${ }^{27}$. Este enfoque permite concentrarse en los aspectos performativos y lingüísticos de la transmisión y comprender el significado del ritual en base precisamente a las múltiples formas atestiguadas a lo largo de su dilatada transmisión.

El análisis de las características particulares de un conjuntos como los Textos de las Pirámides es, sin duda alguna, complejo. El corpus demuestra cierta ambigüedad en la definición del propio concepto -y naturaleza- del ritual y desafortunadamente también desvela los límites de nuestro conocimiento sobre el mismo. Obviamente, la exploración de los aspectos culturales y religiosos en una composición de estas características obliga al investigador a confrontar elementos curiosos, misteriosos o anómalos (p. ej. Topmann 2002 sobre los textos para evitar la necesidad de alimentarse de excrementos en el Más allá, cuyo fundamento performativo y cultural émico no

\footnotetext{
${ }^{26}$ En la misma línea, véanse Michaels 2010, 9-10; Hardin 1983, 846-862.

27 Contra De Buck 1935, xii, al establecer que no había principio que rigiese el orden de los Textos de las Pirámides o Textos de los Ataúdes en las distintas selecciones; también Barta 1981, 69-71; Breasted 1912, 93-94.
} 
está claro $)^{28}$, como indica Michael Carter, quien comenta que «in modern use, ritual often carries connotations of empty, mindless, even neurotic behavior. It is eccentric, set apart from the ordinary, the purposeful, and the meaningful» (Carter 1991, 211). Para muchos autores, la naturaleza diferente del corpus redunda en el pensamiento mítico imbuido en los textos, en la estructura mitológica que encierra. Sin embargo, como hemos visto, estos mismos autores no hallan indicios en los Textos de las Pirámides que les permitan reconstruir una estructura, sentido o argumento narrativo con las sucintas nociones míticas encontradas en el mismo.

Surge entonces la pregunta de si se puede explicar los rituales funerarios en el antiguo Egipto a través de los textos que los representan, sin considerar los componentes mitológicos y más populares (folclóricos, alegóricos), es decir, a través de su propia estructura performativa. En otras palabras, se pretende esclarecer si existe una sintaxis ritual precisa que los sacerdotes y la comunidad reconociera y, si fuese así, si se pueden identificar sus elementos -segmentos rituales, conexiones, principios que regulan las relaciones internas- $-\mathrm{y}$ el enfoque cognitivo que los antiguos egipcios aplicaron a su uso.

\section{SINTAXIS Y SIGNIFICADO RITUAL}

En el análisis de la teoría de Frits Staal, Seaquist observa que éste razona la clara analogía entre la estructura del ritual y la estructura natural del lenguaje (Seaquist 2004, 154-157). El argumento inicial de Staal puede ser aplicado a los datos empíricos del corpus y de este modo reconocer una relación directa entre la estructura compleja del ritual, constituida por numerosos ritos de diversa naturaleza, extensión y significado, y la forma textual de los Textos de las Pirámides, cuyos segmentos o series funcionan aisladamente o en conjunto como un sistema no-narrativo de sucesiones ceremoniales que benefician al difunto en su tumba. Consiguientemente, se puede determinar también el valor de la tesis sintáctica y la gramaticalidad («well-formedness»: Chafe 1970, $346)^{29}$ de los componentes del ritual en ese collage de los Textos de las Pirámides.

Además, con respecto a la trasmisión de los diversos segmentos o grupos de los Textos de las Pirámides así como su comportamiento, este estudio también encara dos cuestiones que Seaquist lanza a historiadores, antropólogos y etnógrafos. Seaquist estima que responder a estas cuestiones requeriría la asistencia de especialistas

\footnotetext{
${ }^{28}$ Como ejemplo, TA 184 ECT III, 79b-e, 79h-80e (ataúd T1L de Imau)

tm wnm(w) hs $m \underline{h r}$ rt-ntrr Para no comer excrementos en la necrópolis,

$\operatorname{tm} \check{s} m(w) m$ shd

shm $m$ mw tzw

pr.t $m$ hrw $[\ldots]$

bw.t $\operatorname{Im} z$ zp $2 n w n m(w)=f \quad$ Imau no comerá aquello que detesta profundamente (lit. dos veces).

bw.t Imзw pn pw hs para no caminar boca abajo,

para adquirir poder sobre el agua y el aire,

y salir durante el día $[\ldots]$

$n w n m=f h t p-k 3 n z m_{3} I m 3 w$ pn jm=f él no comerá excrementos y no se unirá a ello.

${ }^{29}$ En el presente estudio, el concepto gramaticalidad o «well-formedness» implica una correspondencia precisa entre las ceremonias y los textos, resultado de la capacidad del corpus de adaptarse a las tradiciones y prácticas funerarias de cada localidad.
} 
en diferentes tradiciones religiosas. Desde la perspectiva de la teoría del ritual, solo cuando los especialistas en diversas tradiciones respondan se podrá saber cómo de poderosa es esta teoría (Seaquist 2004, 3).

La primera cuestión versa sobre la capacidad sintáctica del ritual. La categorización, segmentación y el uso de múltiples selecciones con Textos de las Pirámides demuestran claramente que se trata de una estructura sintáctica compleja. La segunda cuestión hace referencia a la posibilidad de que el significado del ritual sea composicional (Seaquist 2004, 2-7). Esta cuestión pugna con la idea de Staal según la cual un ritual no presenta un significado concreto. Más que tratar con el problema definicional que sugiere esta idea, es preferible considerar que en los Textos de las Pirámides la sintaxis del ritual contribuye al significado de cada uno de sus componentes y exhibe reglas que gobiernan las series y textos constituyentes. Por ejemplo, al examinar una selección de textos de los Reinos Antiguo y Medio no sólo se identifica la lógica sintáctica de su estructura y segmentos, sino que además se puede reconocer el carácter del programa textual, definido por la aparición de un repertorio tradicional de textos, que denota la preservación de formas tradicionales en el ritual (conservatio), o por la presencia de una selección textual novedosa, es decir, implicando un cambio de significado (mutatio).

Por ejemplo, en la tabla 1 se pueden observar los tipos de programas textuales compuestos para cada monumento o mobiliario (ataúd, sarcófago, caja de ajuar) y comparar la secuencia textual con la versión original del Reino Antiguo. En este caso,

\begin{tabular}{|c|c|c|c|c|c|c|}
\hline \multicolumn{7}{|c|}{ Versión original - Reino Antiguo, dinastía V: pirámide de Unas, Saqqara } \\
\hline \multicolumn{2}{|c|}{$\begin{array}{l}\text { TP247-258, ТP260-263, TP } 267-272 \\
\text { Incorporación a la comunidad divina }\end{array}$} & $\begin{array}{l}\text { TP273-276 } \\
\text { Apotropaia }\end{array}$ & & & & \\
\hline \multicolumn{7}{|c|}{ Programa tradicional - Reino Medio, dinastía XII: tumba de Senwosertankh, Lisht } \\
\hline \multicolumn{2}{|c|}{$\begin{array}{l}\text { TP247-258, TP260-263, TP267-272 } \\
\text { Incorporación a la comunidad divina }\end{array}$} & $\begin{array}{l}\text { TP273-274 } \\
\text { Apotropaia }\end{array}$ & & & & \\
\hline \multicolumn{7}{|c|}{ Programa tradicional - Reino Medio, dinastía XII: tumba de Siese, Dahshur } \\
\hline \multicolumn{2}{|c|}{$\begin{array}{l}\text { TP247-258, ТP260-263, TP267-272 } \\
\text { Incorporación a la comunidad divina }\end{array}$} & $\begin{array}{l}\text { TP273 } \\
\text { Apotropaia }\end{array}$ & & & & \\
\hline \multicolumn{7}{|c|}{ Programa renovador - Reino Medio, dinastía XII: tumba de Imhotep, Lisht } \\
\hline \multicolumn{2}{|c|}{$\begin{array}{l}\text { TP254-258, TP260-263, TP267 + TP270 } \\
\text { Incorporación a la comunidad divina }\end{array}$} & & \multicolumn{3}{|c|}{$\begin{array}{l}\text { TP204-205, TP207, TP209-210 } \\
\text { Entrega de ofrendas al difunto }\end{array}$} & $\begin{array}{l}\text { TP268-272 } \\
\text { Incorporación }\end{array}$ \\
\hline \multicolumn{7}{|c|}{ Programa renovador - Inicios del Primer Período Intermedio, dinastías VII-VIII: tumba de Neha, El-Qatta } \\
\hline \multicolumn{2}{|c|}{$\begin{array}{l}\text { TP270-272 } \\
\text { Incorporación a la comunidad divina }\end{array}$} & & $\begin{array}{l}\text { TP302-304 } \\
\text { Transformación }\end{array}$ & $\begin{array}{l}\approx \text { TA472 } \\
\text { Recitación } \\
\text { al shabti }\end{array}$ & $\begin{array}{l}\text { TP579 } \\
\text { Servicio } \\
\text { a Osiris }\end{array}$ & $\begin{array}{l}\text { TA63-65 } \\
\text { Glorificación }\end{array}$ \\
\hline \multicolumn{7}{|c|}{ Programa renovador - Reino Medio tardío, dinastía XIII: caja de canopos de Hepi-Ankhtify, Meir } \\
\hline $\begin{array}{l}\text { TP249 } \\
\text { Incorporación } \\
\text { a la comunidad divina }\end{array}$ & $\begin{array}{l}\approx \mathrm{TP} 667, \approx \mathrm{TP} 664 \\
\text { Al frente de los } \\
\text { dioses }\end{array}$ & \begin{tabular}{|l|} 
TP677 \\
Lamentos
\end{tabular} & $\begin{array}{l}\approx \mathrm{TP} 637 \\
\text { Ofrendas }\end{array}$ & \multicolumn{2}{|c|}{$\begin{array}{l}\approx \text { TP450, TP433 } \\
\text { Protección de Nut }\end{array}$} & \\
\hline
\end{tabular}

Tabla 1: Sección de rituales para el difunto (programas tradicionales y renovadores) 
la versión de Unas, último rey de la Dinastía V, sirve como referente para entender que las secuencias de Senwosertankh y Siese siguieron los patrones rituales (grupos, posición, orden y función) del arquetipo (Vorlage), mientras que los programas en las tumbas de Imhotep y Neha así como la caja de canopos de Hepi-Ankhtify incorporaron secuencias rituales distintas, lo que implica una reconsideración de la secuencia ritual y quizás cierta especulación teológica.

Hasta ahora se ha discutido la naturaleza performativa intrínseca a los Textos de las Pirámides y, por lo tanto, se ha definido el corpus como un producto social y cultural en forma de collage de textos rituales o «purposive acts that have constitutive functions in a given reality or form of existence» (Gruenwald 2003, 25). Sin embargo, es fundamental discernir los mecanismos esenciales que hacen que esa colección, como concatenación de ritos que se ejecutan y perpetúan en el tiempo para la resurrección y salvación del difunto, adquiera estructura. Además, la funcionalidad que se reconoce en los diversos segmentos de esta concatenación otorga significado a cada uno de sus componentes, a los textos y a las secuencias que constituyen dicha cadena y que juegan un papel singular en la integración de los diversos rituales con un fin común, el bienestar del difunto en el Más Allá ${ }^{30}$.

La ejecución de un ritual activa un proceso dinámico lleno de significado (Jennings 1982, 114-117) cuya finalidad es proporcionar potencia mágica al difunto para el que se ejecuta. En el caso de los Textos de las Pirámides, la función performativa de cada selección inscrita en una pirámide, tumba o ataúd se define por la propia sucesión y por sus constituyentes, es decir, por la estructura y orden que se les asigna y la eficacia conjunta e individual de las recitaciones mágicas de carácter sacerdotal y personal. Por ello, el orden de ritos, los mecanismos que vinculan las distintas cadenas rituales y el propósito mágico de cada texto que las componen son factores del proceso ritual que están intrínsecamente conectados con el resultado final del mismo. Así pues, la fuente más prominente para el discernimiento del significado de un ritual es la estructura sintáctica y las partes constituyentes del proceso sobre el que se fundamentan los beneficios efectivos de dicho ritual para el difunto y su espíritu $a k h$.

Además, un factor adicional en la comprensión del significado del ritual en los Textos de las Pirámides es la propia percepción que los egipcios manifestaron sobre sus ceremonias (émica), tanto en referencias externas como en los propios textos del corpus. De hecho, en el Reino Antiguo los egipcios reflejaron en inscripciones privadas su apreciación por aquellos rituales que aseguraban una experiencia post mór-

\footnotetext{
${ }^{30}$ TP 470 Pir. 914c-917c (Pepi I) $s \underline{d} 3=f \underline{t}$ nj $s \underline{d} 3$ Ppj pn jr p.tn $n h$ w ws nb $m_{3}$ Ppj pn jt=f m3 Ppj pn $R^{\prime} j=j$ jr j3.wt q3j.t jr j3.wt Stš.t rdj sw j3.wt q3j.t n j3.wt Stš.t $n$ nh.t tf q3.t j3bt.t p.t qrqr.tj hms.t ntr.w dp=s $n$ Ppj js pw' $n h$ (Hr) wb3 qbhw
}

¿Dónde irá (el rey Pepi)? «Este Pepi irá al cielo de toda vida y poder, para que este Pepi pueda ver a su padre y pueda ver a Re», digo. (Pepi irá) a las altas cumbres y a las cumbres setianas, y las altas cumbres lo llevarán a las setianas y a aquél sicomoro alto al oriente del firmamento, vibrante, sobre el que los dioses se sientan, porque Pepi es el viviente, Horus, que explora las aguas celestiales. 
tem satisfactoria. Lo interesante de estas inscripciones es su referencia a los rituales representados por los Textos de las Pirámides, lo que refuerza la idea de que estas ceremonias se celebraban regularmente en la comunidad, aunque solamente la miembros de la realeza pudieran contar con inscripciones de los rituales, al menos hasta finales del Reino Antiguo. Posteriormente, en el Reino Medio, cuando los Textos de las Pirámides aparecen ya en las tumbas y ajuares de la elite, sus recitaciones reciben títulos y éstos demuestran que su función (i.e. $s 3$ h.t «transformar en un $a k h$ ») era la misma que la de los rituales celebrados en el contexto privado del Reino Antiguo (i.e. jr.t j.h.t $n b(. t) 3 h$ «realizar todos los actos de un $a k h »)$ :

Inscripción de Ti, Dinastía V, Saqqara

jw jr $n(=j) j . h . t n b(. t) 3 h$ špss jrr.t n jqr jmj $n$ 3h.w $m$ wn.wt hrjj.h.t $n b(. t) 3 h \breve{s} p] s s$

Todo ritual con el que uno se transforma en un noble espíritu $a k h$ ha sido llevado a cabo para mí, (es decir) aquello que se hace para uno excelente entre todos los espíritus akh a través del servicio del sacerdote lector. Yo he sido iniciado en cada rito por el que uno se transforma en un noble espíritu $a k h$ (Edel 1944, 66-67).

Inscripción de Ankhmahor, Dinastía VI, Saqqara

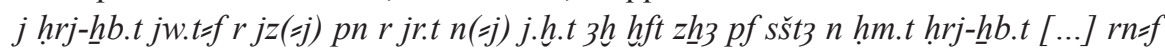
$\check{s} d n(=j) s 3 h{ }^{\circ} p r$

¡Oh sacerdote lector que entre en esta tumba mía para realizar para mí los actos de transformación en un espíritu $a k h$ según las escrituras enigmáticas del oficio de sacerdote lector $[\ldots]$ su nombre, y recite para mí un ensalmo eficaz de transfiguración en $a k h$ (Sethe 1933: Urk. I 202, 15-18).

Ataúd T9C de Mentuhotep, Dinastía XII, Tebas

TP 72, ECT VIII, 50a

rz.wn(j)w mrh.t n(j)w 3 h $m \underline{h}$ r.t-nt $\underline{t} r$

Ensalmos de ungüento de un espíritu akh en la necrópolis (Allen 2006a, 18).

Tumba Sq18X de Ihy, Dinastía XII, Saqqara

TP 77, col. 7

s3h.w r. $n$ mrh.t

Transformaciones en $a k h$. Ensalmo de ungüento (Firth and Gunn 1926, 287).

Ataúd M1C de Rerut, Dinastía XII, Meir

TP 213, ECT VIII, 134a

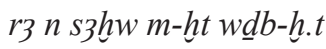

Ensalmo de transformación en espíritu akh tras la reposición de ofrendas (Allen 2006a, 46).

Ataúd L-A1 de Ankhti, Dinastía XII, Lisht

TP 220, ECT VIII, 194a

$\underline{d} d$-mdw s3h.t $m$ h hr.t-ntrr rz.w 'š3.w wnn r-gs nțr m jmn.t 
Recitación. Transformándose en un espíritu akh en la necrópolis. Ensalmos múltiples para estar al lado del dios en el Occidente (Allen 2006a, 192).

En el pasado algunos egiptólogos han intentado responder a cuestiones fundamentales sobre el significado de los rituales en los Textos de las Pirámides y el corpus asociado de los Textos de los Ataúdes, examinando el discurso y temáticas de las recitaciones que los componen. Su intención era la de identificar características singulares a nivel literario que ayudasen a conectar las distintas secuencias o series de textos que constituían y daban forma al corpus. Con ello se pretendía identificar un orden narrativo que sistematizase y diera sentido a la concatenación de grupos de textos de distinta índole. Sin embargo, como se ha explicado anteriormente, la naturaleza ritual de estos textos no propone un discurso narrativo en el cual cada unidad complementa y amplifica una cadena literaria de contenido -narrativo, mítico, lógico- como en el caso de, por ejemplo, un tratado filosófico, una novela o un relato mítico coherente. Al contrario, el significado del ritual es observable a nivel sintáctico, dependiente de la relación de autodefinición de los textos rituales unidos como actos de un discurso esotérico, mágico y soteriológico. En este punto merece la pena referirse a las características internas de cada recitación o al uso de expresiones recurrentes que se repiten constantemente y que permiten distinguir las series y sus textos. Estas características y reiteraciones constituyen atributos particulares a cada serie - contribuyen a definirlas-y funcionan como enlaces internos entre los distintos componentes de una serie. Es lo que Hays y Schenk definieron como «intertextual linkages» (Hays y Schenk 2007, 179-181; Hays 2006, 16-18) y Roland Barthes como «signifié de connotation»(Barthes 1970, 180-182). Estos vínculos en contenido definen los textos de cada serie y los distinguen de otros grupos, pero no obedecen a reglas del discurso literario sino a la propia naturaleza performativa funcional que constituye los Textos de las Pirámides. Precisamente tal mise au point por parte de algunos egiptólogos en la observación del comportamiento del corpus precipitó la interpretación de los textos como composiciones literarias sin considerar la afiliación del texto a un tipo de rito común en la serie, prescindiendo de todo interés en las categorías rituales, la cadena de ejecución y el contexto ritual que la serie representaba.

Sin entrar a cuestionar la presunción del «sin sentido» del ritual de Frits Staal, este estudio aplica su aproximación lingüística a los Textos de las Pirámides y su propuesta de identificar la estructura ritual con el lenguaje. Como sabemos, existen proposiciones similares que expresan la correlación del ritual y el lenguaje en una dimensión sintáctica y rechazan la idea de la naturaleza sin significado de los actos (Penner 1991, 3) ${ }^{31}$. El objetivo de esta discusión, por lo tanto, va más allá de comprender cuáles son las reglas (Staal 1991, 333) y se centra en probar que el análisis semántico de la sintaxis promueve la definición de las series de textos, su relación intertextual y su función como unidades performativas del ritual representado por

\footnotetext{
31 Para una respuesta ambigua de Frits Staal a sus detractores, véase Staal 1991, 227-228.
} 
el corpus. El comportamiento de sus textos y su afiliación a series secuenciadas evidencian el sentido sintáctico del mismo y el valor del significado del ritual.

En base a la contextualización del significado y el rol que cada segmento sintáctico o serie representaba, el lingüista Stephen Ullmann enfatizó la importancia de los términos «nombre», «sentido» y «cosa» para la mejor comprensión de la matriz de un significado (Ullmann 1972, 57; 1951, 69-70). De acuerdo con estos términos, que constituyen los criterios para la «teoría referencial del significado» de Ullmann $(1972,68)$, se puede proponer aislar las nociones «recitación», «contexto» y «rito» en la matriz del significado ritual en los Textos de las Pirámides y conectar la evidencia obtenida del análisis de los textos del corpus con las ideas sintácticas y referenciales de Staal, Ullmann e incluso Jakobson, quien estudia en detalle la contigüidad entre el lenguaje y su función (1956).

Adicionalmente, los ritualistas no han discutido la cuestión del propósito último del ritual desde la perspectiva de sus agentes y participantes. Incluso si Staal llevase razón y fuera el caso que los oficiantes de un ritual (sacerdotes) no experimentasen realmente ningún pensamiento significativo sobre la ejecución y beneficio de un ritual (Staal 1979, 3), habría que aceptar que los participantes se implicasen en el ritual con la esperanza de encontrar una respuesta a cuestiones como la muerte, el Más Allá o la salvación del espíritu (véase esp. Robbins 2001, 593-595). Una respuesta como ésta implica un conocimiento que los oficiantes del ritual dotaban de modo biológico a un fenómeno social, cultural e intelectual que, sin duda alguna, tenía significado religioso e incluso íntimo y personal para los participantes. Los rituales funerarios reunidos en el corpus de los Textos de las Pirámides representaban ceremonias y prácticas mágicas derivadas de siglos de creencias, supersticiones e intentos por comprender lo divino. Estas prácticas, tanto si se trasmitieron oralmente como si fueron entextualizadas y puestas por escrito, eran respetadas fielmente por la comunidad (Hays 2010; Assmann y Kucharek 2008). El objetivo del oficiante era ejecutar cada acto con la esperanza de que beneficiara al difunto (Michaels 2003, 305; 2000, 104-123). Ello implica la comprensión por parte del sacerdote y la comunidad que atiende el evento de la relación existente entre su ejecución y el beneficio que reporta.

Además, la percepción de la transmisión de los Textos de las Pirámides (sobre todo en su primera fase desde el reinado de Unas hasta el fin de la VI Dinastía), como un proceso personalizado en el que cada selección del corpus estaba cuidadosamente preparada de acuerdo con la estructura sacramental del culto de cada rey, reina o tendencia teológica (e.g. tradición estelar, osiriana o solar), también nos muestra que los rituales y los textos poseían un significado propio notable. Las adaptaciones posteriores que se emplean en el contexto de los enterramientos privados también revelan procesos de personalización, influencia de la tradición religiosa local $\mathrm{y}$, en gran medida, la presencia de significado detrás de los textos, sus series y la forma adquirida por el corpus. 


\section{CONCLUSIÓN}

Definir el significado concreto y los mecanismos empleados en los diversos rituales funerarios del Egipto faraónico -así como los textos que los expresaban, ejecutaban y perpetuaban- es fundamental para profundizar en el conocimiento de los aspectos religiosos y sociales más difíciles de rastrear en la cultura material, pero que constituyen, sin duda alguna, la clave para entender las creencias y los comportamientos. En palabras de Schechner refiriéndose al ritual como comportamiento, se trata de indagar sobre un «crux, cross, crossing, decisive meeting place» en el debate sobre una cultura milenaria (Schechner 1985, 195). Por ello, merece la pena considerar el marco teórico y algunas de las cuestiones expuestas por ritualistas involucrados en el estudio de las sociedades antiguas, sus rituales y textos para, de ese modo y desde una perspectiva egiptológica, «recoger el guante» que Seaquist lanza y dejar que los Textos de las Pirámides se conviertan en la evidencia empírica para la teoría.

En este trabajo se ha explorado el corpus de Textos de las Pirámides desde la perspectiva de la sintaxis del ritual. Esta aproximación propone que la estructura semántica del mismo depende de la afiliación o presencia de una serie de grupos o segmentos con integridad y función propias, que históricamente procedían de diversos contextos pero terminaron por ser combinados en una secuenciación compleja cuyo propósito no era otro que favorecer al rey difunto en el Reino Antiguo -más tarde a cualquier individuo que pudiera contar con estos materiales- y reproducir en el espacio íntimo de su pirámide un collage soteriológico sin estructura narrativa pero con una sintaxis ritual profunda y eficaz, un compendio que asegurase por todos los medios conocidos el triunfo del espíritu $a k h$.

\section{REFERENCIAS BIBLIOGRÁFICAS}

P. Ackerman, «Jane Harrison and the Cambridge Ritualists» en P. Ackerman (ed.), The Myth and Ritual School: J.G. Frazer and the Cambridge Ritualists, Garland Publishing, New York-London, 1991.

N. Alexanian, «Tomb and social status. The textual evidence» en M. Bárta (ed.), The Old Kingdom Art and Archaeology. Proceedigs of the Conference. Prague, May 21-June 4, 2004, Academy of Sciences, Prague, 2006.

D. Allen, Structure and Creativity in Religion. Hermeneutics in Mircea Eliade's Phenomenology and New Directions, Mouton Publishers, The Hague, 1978.

J.P. Allen, The Egyptian Coffin Texts: Middle Kingdom copies of Pyramid Texts, vol. 8, University of Chicago Press, Chicago, 2006a.

J.P. Allen, «Some aspects of the non-royal afterlife in the Old Kingdom» en M. Bárta (ed.), The Old Kingdom Art and Archaeology. Proceedigs of the Conference. Prague, May 21June 4, 2004, Academy of Sciences, Prague, 2006b.

J.P. Allen, The Ancient Egyptian Pyramid Texts, SBL Press, Atlanta, 2005. 
J.P. Allen, «Reading a Pyramid» en C. Berger, G. Clerc y N. Grimal (eds.), Hommages à Jean Leclant, vol. 1, IFAO Press, Caire, 1994.

H. Altenmüller, «Zum Ursprung von Isis and Nephthys» en Studien zur Altägyptischen Kultur, vol. 27, H. Buske Verlag, Hamburg, 1999.

H. Altenmüller, «Zur Ritualstruktur der Pyramidentexte» en ZDMG Supplement, vol. 2, F. Steiner Verlag, Wiesbaden, 1974.

H. Altenmüller, Die Texte zum Begräbnisritual in den Pyramiden des Alten Reiches, Otto Harrassowitz, Wiesbaden, 1972.

D.A. Anderson, «Evidence for early ritual activity in the Predynastic settlement at el-Mahâsna» en R.F. Friedman y P.N. Piske (eds.), Egypt at its Origins 3, Peeters, Leuven, 2011.

R. Anthes, «Egyptian Theology in the Third Millennium B.C. » en Journal of Near Eastern Studies, vol. 18.3, University of Chicago Press, Chicago, 1954.

J. Assmann, Ägyptische Geheimnisse, Wilhelm Fink, München, 2004.

J. Assmann, Religion und kulturelles Gedächtnis. Zehn Studien, C.H. Beck, 2000.

J. Assmann, «Egyptian Mortuary Liturgies» en S. Israelit-Groll (ed.), Studies in Egyptology Presented to Miriam Lichtheim, vol. 1, Magnes Press, Jerusalem, 1990.

J. Assmann, «Die Verborgenheit des Mythos in Ägypten» en Göttinger Miszellen, vol. 25, Universität Göttingen, Göttingen, 1977.

J. Assmann y A. Kucharek, Ägyptische Religion. Totenliteratur, Verlag der Weltreligionen, Frankfurt am Mein-Leipzig, 2008.

J. Baines, «Aesthetic Culture and the Emergence of Writing in Egypt during Naqada III» en Archéo-Nil, vol. 20, Sociedad Archéo-Nil, Paris, 2010.

J. Baines, «Modelling Sources, Processes, and Locations of Early Mortuary Texts» en S. Bickel y B. Mathieu (eds.), D'un monde à l'autre. Textes des Pyramides \& Textes des Sarcophages, IFAO Press, Caire, 2004.

J. Baines, «Forerunners of narrative biographies» en A. Leahy y W.J. Tait (eds.), Studies on Ancient Egypt in Honour of H.S. Smith, EES, London, 1999a.

J. Baines, «Prehistories of literature: performance, fiction, myth» en G. Moers (ed.), Definitely: Egyptian Literature, Hubert \& Co, Göttingen, 1999b.

J. Baines, «Kingship, Definition of Culture, and Legitimation» en D. O'Connor y D.P. Silverman (eds.), Ancient Egyptian Kingship, Brill, Leiden-New York-Köln, 1995.

J. Baines, «On the Status and Purposes of Ancient Egyptian Art» en Cambridge Archaeological Journal, vol. 4.1, Cambridge University Press, Cambridge, 1994.

J. Baines, «Restricted Knowledge, Hierarchy and Decorum: Modern Perceptions and Ancient Institutions» en Journal of Near Eastern Studies, vol. 50.2, University of Chicago Press, Chicago, 1991.

J. Baines, «Egyptian Myth and Discourse: Myth, Gods, and the Early Written and Iconographic Record» en Journal of American Research Center in Egypt, vol. 27, ARCE, Boston, 1990.

J. Baines, «Communication and Display: the Integration of Early Egyptian Art and Writing» en Antiquity, vol. 63.240, Cambridge University Press, York, 1989. 
E.J. Bakker, «Activation and Preservation: The Interdependence of Text and Performance in an Oral Tradition» en Oral Tradition, vol. 8.1, Slavica, Columbus, 1993.

W. Barta, Die Bedeutung der Pyramidentexte für den verstorbenen König, Deutscher Kunstverlag, München-Berlin, 1981.

R. Barthes, S/Z, Éditions du Seuil, Paris, 1970.

C. Bell, Ritual: Perspectives and Dimensions, Oxford University Press, Oxford, 1997.

S. Bickel, La cosmogonie égyptienne avant le Nouvel Empire, Vandenhoeck\&Ruprecht, Fribourg-Göttingen, 1994.

N. Billing, «Monumentalizing the beyond: reading the pyramid before and after the Pyramid Texts» en Studien zur Altägyptischen Kultur, vol. 40, H. Buske Verlag, Hamburg, 2011.

C.J. Bleeker, «The Contribution of the Phenomenology of Religion to the Study of the History of Religions» en U. Bianchi, C.J. Bleeker y A. Bausani (eds.), Problems and Methods of the History of Religions, Brill, Leiden, 1972.

J.H. Breasted, The Dawn of Conscience, Charles Scribner's Sons, New York, 1933.

J.H. Breasted, Development of Religion and Thought in Ancient Egypt, Charles Scribner's Sons, New York, 1912.

J.M. Bremmer, "'Religion', 'ritual' and the opposition 'sacred vs profane'. Notes towards a terminological 'genealogy'» en F. Graf (ed.), Ansichten grieschischer Rituales. Geburtstagssymposium für Walter Burkert, B.G. Teubner, Stuttgart-Leipzig, 1998.

A. De Buck, The Egyptian Coffin Texts I-VII, University of Chicago Press, Chicago, 19361961.

M. Campagno, «Space and Shape: Notes on Pre- and Proto-state Funerary Practices in Ancient Egypt» en S. Bickel y A. Loprieno (eds.), Basel Egyptology Prize 1. Junior Research in Egyptian History, Archaeology, and Philology, Schwabe \& Co. Ag. Verlag, Basel, 2003.

M.F. Carter, «The Ritual Functions of Epideictic Rhetoric: The Case of Socrates's Funeral Oration» en Rhetorica, vol. 9, University of California Press, Berkeley, 1991.

W.L. Chafe, Meaning and the Structure of Language, University of Chicago Press, Chicago, 1970 .

N. Chomsky, Syntactic Structures, Mouton, The Hague, 1957.

R. David, Religion and Magic in Ancient Egypt, Penguin, London, 2002.

W.G. Doty, Mythography. The Study of Myths and Rituals, University of Alabama Press, Alabama, 1986.

E. Edel, «Untersuchungen zur Phraseologie der ägyptischen Inschriften des Alten Reiches» en Mitteilungen des Deutschen Instituts für Ägyptische Altertumskunde in Kairo, vol. 13, Harrassowitz, Wiesbaden, 1944.

M. Eliade, Patterns in Comparative Religion, Sheed \& Ward, London, 1957.

C.J. Eyre, The Cannibal Hymn. A Cultural and Literary Study, Liverpool University Press, Liverpool, 2002.

C.M. Firth y B. Gunn, Teti Pyramid Cemeteries, vol. 1, IFAO Press, Caire, 1926.

A. Van Gennep, The Rites of Passage, University of Chicago Press, Chicago, 1960.

R.A. Gillam, Performance and drama in ancient Egypt, Duckworth, London, 2005. 
K. Goebs, «A Functional Approach to Egyptian Myth and Mythemes» en Journal of Ancient Near Eastern Religion, vol. 2, Brill, Leiden, 2003.

O. Goldwasser, «The Narmer Palette and the 'Triumph of Metaphor'» en Lingua Aegyptia, vol. 2, Seminar für Ägyptologie und Koptologie, Göttingen, 1992.

J. Goody, «Against 'ritual': Loosely structured thoughts on a loosely defined topic» en P.J. Stewart y A. Strathen (eds.), Ritual, Ashgate Publishing, Farnham, 2010.

A.G. Grappard, «Rule-governed Activity vs. Rule-creating Activity» en Religion, vol. 21, Academic Press, London, 1991.

A.J. Greimas, Structural semantics. An Attempt at a Method, University of Nebraska Press, Lincoln-London, 1983.

I. Gruenwald, Rituals and Ritual Theory in Ancient Israel, Brill, Leiden-Boston, 2003.

W.M. Hanks, «The Indexical Ground of Deictic Reference» en A. Duranti y C. Goodwin (eds.), Rethinking Context. Language as an Interactive Phenomenon, Cambridge University Press, Cambridge, 1992.

R.F. Hardin, «'Ritual' in Recent Criticism: The Elusive Sense of Community» en Proceedings of the Modern Language Association of America, vol. 98.5, Kraus Reprint, New York, 1983.

H.M. Hays, «The end of rites of passage and a start with ritual syntax in ancient Egypt» en C. Ambos y L. Verderame (eds.), Approaching rituals in ancient cultures: Questioni di Rito: Rituali come Fonte di Conoscenza delle Religioni e delle Concezioni del Mondo nelle Culture Antiche, Fabrizio Serra, Pisa, 2013.

H.M. Hays, The Organization of the Pyramid Texts: Typology and Disposition, Brill, BostonLeiden, 2012.

H.M. Hays, «The Death of Democratization of the Afterlife» en N. Strudwick y S. Strudwick (eds.), Old Kingdom, New Perspectives. Egyptian Art and Archaeology 2750-2150, Oxbow Books, Oxford, 2011.

H.M. Hays, «Funerary Rituals (Pharaonic Period)» en J. Dieleman y W. Wendrich (eds.), UCLA Encyclopedia of Egyptology, UCLA Press, Los Angeles, 2010.

https://escholarship.org/uc/item/1r32g9zn [acceso: 25.06.2015]

H.M. Hays, «Old Kingdom Sacerdotal Texts» en Jaarbericht van het Voor-aziatisch Egyptisch Gezelschap «Ex Oriente Lux», vol. 41, Brill, Leiden, 2009.

H.M. Hays, The Typological Structure of the Pyramid Texts and Its Continuities with Middle Kingdom Mortuary Literature, tesis doctoral de la University of Chicago, Chicago, 2006.

H.M. Hays y W. Schenk, «Intersection of Ritual Space and Ritual Representation: Pyramid Texts in Eighteenth Dynasty Theban Tombs» en P.F. Dorman y B.M. Bryan (eds.), Sacred Space and Sacred Function in Ancient Thebes, Oriental Institue-University of Chicago Press, Chicago, 2007.

J.E. Hellum, «Toward an Understanding of the Use of Myth in the Pyramid Texts» en Studien zur Altägyptischen Kultur, vol. 43, H. Buske Verlag, Hamburg, 2014.

J.E. Hellum, «The Use of Myth in the Pyramid Texts» en C.M. Knobaluch y J.C. Hill (eds.), Egyptology in Australia and New Zealand 2009, Archaeopress, Oxford, 2012. 
J.E. Hellum, The Presence of Myth in the Pyramid Texts, tesis doctoral de la University of Toronto, Toronto, 2001.

J.K. Hoffmeier, «Are there regionally-based theological differences in the Coffin Texts?» en H.O. Willems (ed.), The World of the Coffin Texts. Proceedings of the Symposium held on the Occasion of the 100th Birthday of Adriaan de Buck, NINO Press, Leiden, 1996.

E. Hornung, The Ancient Egyptian Books of the Afterlife, Cornell University Press, Ithaca, 1999.

C. Humphrey y J. Laidlaw, «What kind of theory do we need?» en C. Humphrey y J. Laidlaw (eds.), The Archetypal Actions of Ritual. A Theory of Ritual Illustrated by the Jain Rite of Workship, Clarendon Press, Oxford, 1994.

R. Jakobson, «Two aspects of language and two types of aphasic disturbance» en R. Jakobson y M. Halle (eds.), Fundamentals of Language, Mouton, The Hague, 1956.

T.W. Jennings, «On Ritual Knowledge» en The Journal of Religion, vol. 62.2, University of Chicago Press, Chicago, 1982.

J. Kahl, Siut-Theben: zur Wertschätzung von Traditionen im alten Ägypten, Brill, Leiden, 1999.

L. Kákosy, «The Pyramid Texts and Society in the Old Kingdom» en Annales Universitatis Scientarum Budapestinensis de Rolando Eotvos Nominatae. Sectio Historica, vol. 4, Eötvös Lórand, Budapest, 1962.

H. Kees, «Pyramidentexte» en H. Brunner (ed.), Handbuch der Orientalistik, vol. 1.2, Brill, Leiden, 1952.

G.S. Kirk, «On defining Myths» en E.N. Lee, A.P. Mourelatos y R.M. Morty (eds.), Exegesis and Argument: Studies in Greek Philosophy presented to Gregory Vlastos, Van Gorcum, Assen, 1973.

G.S. Kirk, Myth. Its Meaning and Functions in Ancient and Other Cultures, University of California Press, Berkeley-Los Angeles, 1970.

G.A. Klingbeil, Bridging the Gap. Ritual and Ritual Texts in the Bible, Eisenbrauns, Winona Lake, 2007.

E.C. Köhler, «Ursprung einer lange Tradition: Grab und Totenkult in der Frühzeit» en H. Guksch, E. Hofmann y M. Bommas (eds.), Grab und Totenkult im Alten Ägypten, C.H. Beck, München, 2003.

J. Kreinath, J.A.M. Snoek y M. Stausberg, «Ritual Studies, Ritual Theory, Theorizing Rituals - An Introductory Essay» en J. Kreinath, J.A.M. Snoek y M. Stausberg (eds.), Theorizing Rituals: Issues, Topics, Approaches, Concepts, Brill, Leiden, 2006.

B. Mathieu, «La distinction entre Textes des Pyramides et Textes des Sarcophages est-elle légitime?» en S. Bickel y B. Mathieu (eds.), D’un monde à l'autre. Textes des Pyramides \& Textes des Sarcophages, IFAO Press, Caire, 2004.

$\mathrm{B}$. Menu, «Mise à port cérémonielle et prélèvements royaux sous la 1ère Dynastie (NârmerDen)» en Archéo-Nil, vol. 11, Sociedad Archéo-Nil, Paris, 2001.

S.A.B. Mercer, Literary Criticism of the Pyramid Texts, Luzac \& Company, London, 1956.

A. Michaels, «The Grammar of Rituals» en A. Michaels et alii (eds.), Grammars and Morphologies of Ritual Practices in Asia, Harrassowitz, Wiesbaden, 2010. 
A. Michaels, Zur Dynamik von Ritualkomplexen, Universität Heidelberg, Heidelberg, 2003.

A. Michaels, «Ex opere operato: zur Intentionalität promissorischer Akte in Ritualen» en K.P. Köpping y U. Rao (eds.), Im Rausch des Rituals. Gestaltung und Transformation der Wirklichkeit in körperlicher Performanz, Lit Verlag, Münster, 2000.

M.A. Molinero Polo, «Realeza y concepción del universo en los Textos de las Pirámides» en Boletín de la Sociedad Española de Ciencias de las Religiones, vol. 12, SECR, Madrid, 1999.

M.A. Molinero Polo et alii, «Textos de las Pirámides de la Dinastía XXV: Estudio Textual e Histórico Preliminar» en L.M. de Araujo y J. das Candeias Sales (eds.), Novos trabalhos de Egiptologia Ibérica: IV Congresso Ibérico de Egiptología, vol. 2, Universidade de Lisboa, Lisboa, 2012.

A.J. Morales, «Unraveling the Thread. Transmission and Reception of Pyramid Texts in Late Period Egypt» en S. Bickel y L. Díaz-Iglesias (eds.), Studies in Ancient Egyptian Funerary Literature, Peeters, Leuven, 2016a.

A.J. Morales, «From voice to papyrus to wall: Verschriftung and Verschriftlichung in the Old Kingdom Pyramid Texts» en M. Hilgert (ed.), Understanding Material Cultures. A Multidisciplinary View, De Gruyter, Berlin, 2016b.

A.J. Morales, «Los dos cuerpos del rey: cosmos y política de la monarquía egipcia» en F. Lozano, P. Giménez y C. Alarcón (eds.), Reyes y dioses: la realeza divina en las sociedades antiguas, Universidad de Huelva, Huelva, 2016c.

A.J. Morales, «Iteration, Innovation und Dekorum» en Zeitschrift für Ägyptische Sprache und Altertumskunde, vol. 142.1, De Gruyter, Leipzig, 2015a.

A.J. Morales, «Tracing Middle Kingdom Pyramid Texts traditions at Dahshur» en G. Miniaci y W. Grajetzki (eds.), The World of Middle Kingdom Egypt (2000-1550 BC), Golden House Publications, London, 2015b.

A.J. Morales, The transmission of the Pyramid Texts into the Middle Kingdom: Philological aspects of a continuous tradition in Egyptian mortuary literature, tesis doctoral de la University of Pennsylvania, Philadelphia, 2013.

A. Moret, «L'accession de la plèbe égyptienne aux droits religieux et politiques sous le Moyen Empire» en Recueil d'études égyptologiques dédiées à la mémoire de Jean-François Champollion, Librairie Ancienne Honoré Champion, Paris, 1922.

K. Nordh, Aspects of Ancient Egyptian Curses and Blessings, Boreas, Uppsala, 1996.

H.H. Penner, «You don't read a myth for information» en N.K. Frankenberry (ed.), Radical Interpretation in Religion, Cambridge University Press, Cambridge, 2002.

H.H. Penner, «Language, Ritual, and Meaning» en Numen, vol. 32, Brill, Leiden, 1991.

N. Picardo, ««Semantic Homicide» and the So-called Reserve Heads: The Theme of Decapitation in Egyptian Funerary Religion and Some Implications for the Old Kingdom» en Journal of the American Research Center in Egypt, vol. 43, ARCE, Boston, 2007.

J.G. Platvoet, «Ritual in Plural and Pluralistic Societies. Instruments for Analysis» en J.G. Platvoet y K. van der Toorn (eds.), Pluralism and Identity. Studies in Ritual Behavior, Brill, Leiden-New York-Köln, 1995. 
J.G. Platvoet, «The Study of Rites in The Netherlands» en Nederlands Theologisch Tijdschrift, vol. 37.3, Veenman \& Zonen, Wageningen, 1983.

R.A. Rappaport, Ritual and Religion in the Making of Humanity, Cambridge University Press, Cambridge, 1999.

R.K. Ritner, The Mechanics of Ancient Egyptian Magical Practice, Oriental InstituteUniversity of Chicago Press, Chicago, 1997.

J. Robbins, «Ritual Communication and Linguistic Ideology: A Reading and Partial Reformulation of Rappaport's Theory of Ritual» en Current Anthropology, vol. 42.5, University of Chicago, Chicago, 2001.

H. Roeder, «Zwischen den Stühlen. Zugangsbeschreibungen zur altägyptischen Religion zwischen Transdisziplinarität und Eigenbegrifflichkeit» en A. Verbovsek, B. Backes y C. Jones (eds.), Methodik und Didaktik in der Ägyptologie. Herausforderungen eines kulturwissenschaftlichen Paradigmenwechsels in den Altertumswissenschaften, Wilhelm Fink, München, 2011.

H. Roeder, «Rituelle Texthandlungsklassen und Ritualdefinition aus altägyptischer Perspektive» en B. Dücker y H. Roeder (eds.), Rituelle Texthandlungsklassen. Interdisziplinäre Betrachtungen zum Verhältnis von Text und Ritual, Forums Ritualdynamik, Heidelberg, 2004.

A.M. Roth, «Social change in the Fourth Dynasty: the spatial organization of pyramids, tombs, and cemeteries» en Journal of the American Research Center in Egypt, vol. 30, ARCE, Boston, 1993.

B. Russo, «Funerary Spells at Saqqara South. Some Considerations about the Inscriptions of Anu's Coffin (Sq20X)» en Zeitschrift für Ägyptische Sprache und Altertumskunde, vol. 139.1, De Gruyter, Leipzig, 2012.

R. Schechner, «Victor Turner's Last Adventure» en Anthropologica N.S., vol. 27.1-2, Saint Paul University, Otawa, 1985.

K.L. Schmitz, «Ritual elements in community» en Religious Studies, vol. 17, Cambridge University Press, London, 1981.

S. Schott, «Bemerkungen zum altägyptischen Pyramidenkult» en H. Ricke (ed.), Beiträge zur ägyptischen Bauforschung und Altertumskunde, Schweizerisches Institut, Cairo, 1950.

S. Schott, Mythe und Mythenbildung im alten Ägypten, J.C. Hinrichs, Leipzig, 1945.

C.A. Seaquist, Ritual Syntax, tesis doctoral de University of Pennsylvania, Philadelphia, 2004.

R.A. Segal, «Myth and Ritual» en J. Kreinath, J. Snoek y M. Stausberg (eds.), Theorizing Rituals: Issues, Topics, Approaches, Concepts, Brill, Leiden, 2006.

R.A. Segal, «The Myth-Ritualist Theory of Religion» en Journal of the Scientific Study of Religion, vol. 19.2, Society SSR, New Haven, 1980.

K. Sethe, Urkunden des Alten Reichs, J.C. Hinrichs, Leipzig, 1933.

K. Sethe, Totenliteratur der alten Ägypter: die Geschichte einer Sitte, Akademie der Wissenschaften, Berlin, 1931.

K. Sethe, Die altaegyptische Pyramidentexte, J.C. Hinrichs'sche Buchhandlung, Leipzig, 19081922.

D.P. Silverman, «Text and Image and the Origin of Writing in Ancient Egypt» en D.C. Patch (ed.), Dawn of Egyptian Art, Yale University Press, New Haven, 2011. 
D.P. Silverman, «Coffin Texts from Bersheh, Kom el Hisn, and Mendes» en H.O. Willems (ed.), The World of the Coffin Texts. Proceedings of the Symposium held on the Occasion of the 100th Birthday of Adriaan de Buck, NINO Press, Leiden, 1996.

D.P. Silverman, «The Nature of Egyptian Kingship» en D. O’Connor y D.P. Silverman (eds.), Ancient Egyptian Kingship, Brill, Leiden-New York-Köln, 1995.

D.P. Silverman, «Textual Criticism in the Coffin Texts» en J.P. Allen et alii (eds.), Religion and Philosophy in Ancient Egypt, Yale University Press, New Haven, 1989.

D.P. Silverman, The Tomb Chamber of \#sw the Elder: The Inscribed Material at Kom el-@ isn, vol. 1, Eisenbrauns, Winona Lake, 1988.

M. Smith, «Democratization of the Afterlife» en J. Dieleman y W. Wendrich (eds.), UCLA Encyclopedia of Egyptology, UCLA Press, Los Angeles, 2009.

http://escholarship.org/uc/item/70g428wj [acceso: 10.04.2015]

J.A.M. Snoek, «Defining 'Rituals'» en J. Kreinath, J.A.M. Snoek y M. Stausberg (eds.), Theorizing Rituals: Issues, Topics, Approaches, Concepts, Brill, Leiden, 2006.

J. Spiegel, Das Auferstehungsritual der Unas-Pyramide. Beschreibung und erlätuerte Übersetzung, Harrassowizt, Wiesbaden, 1971.

J. Spiegel, «Das Auferstehungsritual der Unaspyramide» en Annales du Service des Antiquités de l'Égypte, vol. 53, IFAO Press, Cairo, 1956.

J. Spiegel, «Die religionsgeschichtliche Stellung der Pyramidentexte» en Orientalia, vol. 22.2, Istituto Biblico Pontificio, Roma, 1953.

F. Staal, «Ritual Order» en A.M. Blondeau y M. Schipper (eds.), Essais sur les Rituel III. Colloque du Centenaire de la Section des Sciences Religieuses de EPHE, Peeters, LouvainParis, 1995.

F. Staal, «Without Ritual, About Ritual and Beyond» en Religion, vol. 21, Academic Press, London, 1991.

F. Staal, Rules without meaning: ritual mantras, and the human sciences, Peter Lang, New york, 1989.

F. Staal, «Rites that Make no Sense» en J.S. Yadara y V. Gautam (eds.), The Communication of Ideas, Concept Publishing, New Delhi, 1981.

F. Staal, «Ritual Syntax» en M. Nagatomi et alii (eds.), Sanskrit and Indian Studies. Essays in Honor of Daniel H.H. Ingalls, D. Reidel Publishing, Dordrecht-Boston-London, 1980.

F. Staal, «The Meaninglessness of Ritual» en Numen, vol. 26, Brill, Leiden, 1979.

D. Stewart, «On defining myth: comparisons of myth theory from an Egyptological viewpoint» en H. Abd el Gawad et alii (eds.), Current Research in Egyptology 2011, Durham University 2011, Oxbow Books, Oxford-Oakville, 2012.

I. Strenski, «The Rise of Ritual and the Hegemony of Myth: Sylvain Lévi, the Durkheimians, and Max Müller» en L.L. Patton y W. Doniger (eds.), Myth and Method, University Press of Virginia, Charlottesville, 1996.

I. Strenski, «What's a Rite? Evolution, Exchange and the Big Picture. Review of Rules without Meaning by Frits Staal» en Religion, vol. 21, Academic Press, London, 1991.

S.J. Tambiah, A performative approach to ritual, Oxford University Press, Oxford, 1979.

'Ilu. Revista de Ciencias de las Religiones

2015, 20, 137-164 
V. Tamorri, «Manipulated corpses in Predynastic Egyptian tombs: deviant or normative practices?» en H. Abd el Gawad et alii (eds.), Current Research in Egyptology 2011, Durham University 2011, Oxbow Books, Oxford-Oakville, 2012.

V.A. Tobin, «Myth and Politics in the Old Kingdom of Egypt» en Bibliotheca Orientalis, vol. 49.5-6, NINO Press, Leiden, 1992.

D. Topmann, Die «Abscheu»-Sprüche der altägyptischen Sargtexte. Untersuchungen zu Textemen und Dialogstrukturen, Harrassowitz, Wiesbaden, 2002.

S. Ullmann, Semantics: An Introduction to the Science of Meaning, Basil Blackwell, Oxford, 1972.

S. Ullmann, The Principles of Semantics, Jackson, Son \& Company, Glasgow, 1951.

M. Valloggia, Balat I: Le mastaba de Medou-Nefer I-II, IFAO Press, Caire, 1986.

H. Te Velde, «The History of the Study of Ancient Egyptian Religion and its Future» en Z. Hawass (ed.), Egyptology at the Dawn of the Twenty-First Century, vol. 2, American University in Cairo Press, Cairo, 2003.

F. Weitzel, Die Gestaltung der Lebensfeiern. Die neue Gemeinschaft. Das Parteiarchiv für nationalsozialistische Feier-und Freizeitgestaltung, NSDAP, Berlin, 1942.

H.O. Willems, “"Demokratisierung des Jenseitsglaubens” von späten Alten Reich bis zur Zweiten Zwischenzeit» en J. Assmann y H. Roeder (eds.), Handbuch der altägyptischen Religion, Brill, Leiden, en prensa.

H.O. Willems, Historical and archaeological aspects of Egyptian funerary culture. Religious ideas and ritual practice in Middle Kingdom elite cemeteries, Brill, Leiden, 2014.

J. Zeidler, «Zur Frage der Spätenstehung des Mythos in Ägypten» en Göttinger Miszellen, vol. 132, Universität Göttingen, Göttingen, 1993. 